\section{Empirical embolisation for intermittent spontaneous muscle haemorrhage associated with anticoagulation therapy}

As more patients are receiving anticoagulant therapy, spontaneous muscle haemorrhage (SMH) has become a common, potentially life-threatening complication. ${ }^{12}$ However, no standardised approach for SMH has established and intermittent $\mathrm{SMH}$ is challenging to manage because of lack of reports.

A 77-year-old man with a history of double mechanical heart valve replacement and chronic atrial fibrillation on warfarin complained of sudden onset of painful swelling in the left leg. Contrast-enhanced computed tomography (CT) revealed femoral and crural muscle haemorrhage (figure 1A) despite a moderate intensity of anticoagulation (Prothrombin time-international normalised ratio 2.01). Following blood transfusion for progressive anaemia, urgent angiography including selective angiography demonstrated no evidence of active bleeding. On the following day, he again experienced sudden worsening of painful swelling in the left leg and anaemia, with haemoglobin dropping from 9.8 to $7.0 \mathrm{mg} / \mathrm{dL}$. Since contrast-enhanced CT revealed enlargement of the muscle haematoma with active bleeding (figure 1B), we repeated angiography. Selective angiography still demonstrated no evidence of contrast agent extravasation (figure 2A, B, Supplementary videos A, B). Thus, we made a diagnosis of intermittent SMH probably because of the tamponade effect of the haematoma. Considering the risk of anticoagulation suspension, warfarin was maintained, and further treatment beyond conservative management was needed to prevent recurrent bleeding and progression of the haematoma that can potentially cause disseminated intravascular coagulation. Given this patient's haemodynamic stability, ${ }^{3}$ we attempted empiric embolisation of the arterial branches supplying the haematoma based on CT findings. We embolised an absorbable gelatin sponge through a microcatheter into those branches although permanent embolisation with coil or N-butyl-2-cyanoacrylate is a good alternative. ${ }^{2}$ Completion angiography revealed the occlusion of those branches (figure 2C, D). Three weeks after the
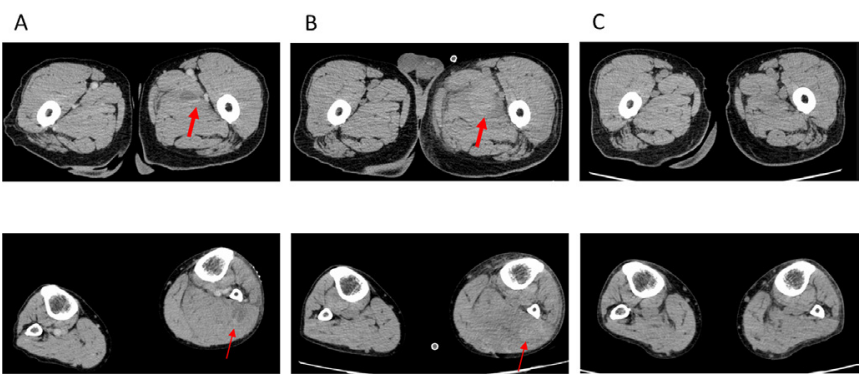

Figure $1 \mathrm{CT}$ of the lower limbs (upper panels, thigh; lower panels, leg). (A) On presentation, Contrast-enhanced CT demonstrated a muscle haematoma with active bleeding from branches of the deep femoral (large arrow) and anterior tibial arteries (small arrow). (B) Although selective angiography revealed no evidence of contrast agent extravasation, contrast-enhanced CT on the following day demonstrated enlargement of the haematoma with active bleeding. (C) Follow-up simple CT after empirical embolisation demonstrated resolution of the haematoma.
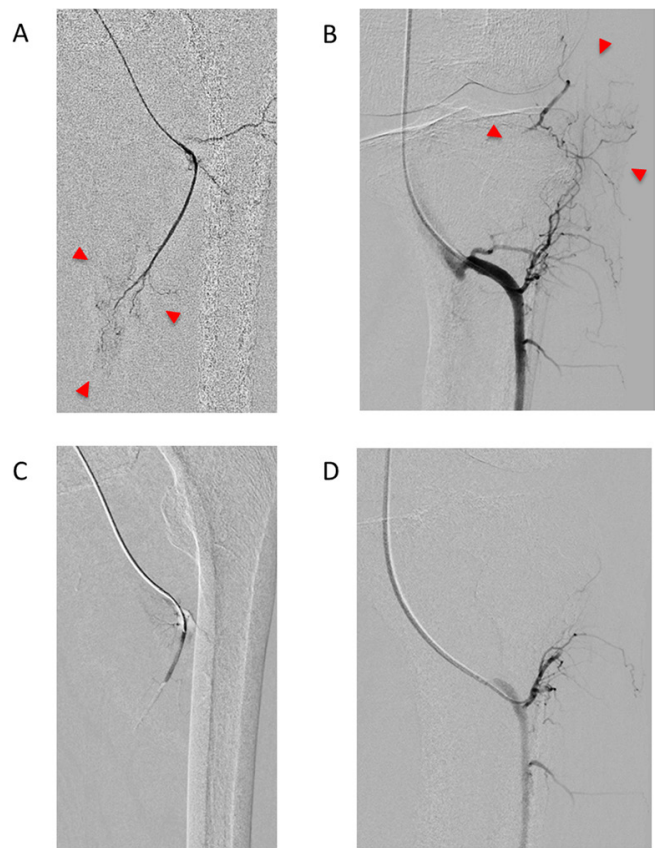

Figure 2 Empirical embolisation of a branch of the left deep femoral artery and a recurrent branch of the left anterior tibial artery. Selective angiography in the perforating branch of the deep femoral artery (A) and the recurrent branch of the anterior tibial artery (B) demonstrated no evidence of contrast agent extravasation (arrowheads). Confirmatory angiography following embolisation demonstrated occlusion of the perforating branch of the deep femoral artery $(C)$ and the recurrent branch of the anterior tibial artery (D).

procedure, the patient was uneventfully discharged with evidence of haematoma resolution (figure 1C).

An awareness of intermittent SMH and empirical embolisation, defined as endovascular occlusion of arteries without visible contrast extravasation on digital subtraction angiography, might contribute to improve patient care. Contrast-enhanced CT might be a good tool to perform effective embolisation.

\section{Kenichiro Sawada, Osami Kawarada, Satoshi Yasuda}

Department of Cardiovascular Medicine, National Cerebral and Cardiovascular Center, Osaka, Japan

Correspondence to Dr Osami Kawarada, Department of Cardiovascular Medicine, National Cerebral and Cardiovascular Center, Osaka 565-8565 Japan; kawarada90@ hotmail.com

Contributors KS and OK were involved in the clinical management of the patient. KS drafted and OK and SY revised the manuscript.

Funding This research received no specific grant from any funding agency in the public, commercial or not-for-profit sectors.

Competing interests None declared.

Patient consent Guardian consent obtained.

Provenance and peer review Not commissioned; externally peer reviewed.

(c) Article author(s) (or their employer(s) unless otherwise stated in the text of the article) 2018. All rights reserved. No commercial use is permitted unless otherwise expressly granted.

- Additional material is published online only. To view please visit the journal online (http://dx.doi.org/10.1136/heartasia-2017-010967).

Check for updates

To cite Sawada K, Kawarada 0, Yasuda S. Heart Asia 2018;10:1-2. 
Heart Asia 2018;10:1-2. doi:10.1136/heartasia-2017-010967

\section{REFERENCES}

1 Dohan A, Sapoval M, Chousterman BG, et al. Spontaneous soft-tissue hemorrhage in anticoagulated patients: safety and efficacy of embolization. AJR Am J Roentgenol 2015;204:1303-10.
2 Ozyer U. Transcatheter arterial embolization with N-butyl-2-cyanoacrylate in the management of spontaneous hematomas. Cardiovasc Intervent Radiol 2017:40:41-9.

3 Popov M, Sotiriadis C, Gay F, et al. Spontaneous intramuscular hematomas of the abdomen and pelvis: a new multilevel algorithm to direct transarterial embolization and patient management. Cardiovasc Intervent Radiol 2017; 40:537-45. 